\title{
ERRATUM
}

Harry H. Schomberg • Dinku M. Endale

\section{Cover crop effects on nitrogen mineralization and availability in conservation tillage cotton}

Published online: 30 November 2004

C) Springer-Verlag 2004

\section{Biol Fertil Soils (2004) Dol 10.1007/s00374-004- 0795-6}

The data for cotton biomass, total N, and LAI in Tables 1 and 2 were incorrect. The correct tables are given here. 
Table 1 Mean above-ground biomass, total $\mathrm{N}$ accumulated in above-ground biomass, LAI, plant height and number of nodes during the 1997 cotton-growing season following crimson clover and rye cover crops

\begin{tabular}{|c|c|c|c|c|c|c|c|c|c|c|}
\hline \multirow{3}{*}{$\begin{array}{l}\text { Cover } \\
\text { Crop }\end{array}$} & \multirow[t]{3}{*}{$\mathrm{DAP}^{\mathrm{a}}$} & \multicolumn{2}{|c|}{ Biomass $^{b}$} & \multicolumn{2}{|c|}{ Total $\mathrm{N}^{\mathrm{b}}$} & \multirow{2}{*}{$\begin{array}{l}\mathrm{LAI}^{\mathrm{b}} \\
\overline{\mathrm{m}^{2} \mathrm{~m}^{-2}}\end{array}$} & \multicolumn{2}{|c|}{ Height } & \multicolumn{2}{|c|}{ Nodes } \\
\hline & & $\mathrm{Mg} \mathrm{h}$ & $a^{-1}$ & $\mathrm{~kg} \mathrm{ha}^{-}$ & & & $\mathrm{cm}$ & & \# & \\
\hline & & & std & & std & std & & std & & Std \\
\hline Clover & 51 & 0.7 & 0.2 & 19.9 & 6.9 & $\begin{array}{lll}0.7 & 0.2\end{array}$ & 43.2 & 3.4 & 9.0 & 1.2 \\
\hline Rye & 51 & 0.8 & 0.2 & 26.6 & 7.5 & $\begin{array}{lll}0.8 & 0.1\end{array}$ & 47.9 & 6.2 & 10.6 & 0.6 \\
\hline Clover & 59 & 1.2 & 0.3 & 32.7 & 11.8 & $\begin{array}{lll}1.1 & 0.3\end{array}$ & 53.2 & 6.0 & 11.6 & 0.7 \\
\hline Rye & 59 & 1.2 & 0.4 & 30.6 & 11.6 & $\begin{array}{ll}1.2 & 0.4\end{array}$ & 58.0 & 7.3 & 11.4 & 1.5 \\
\hline Clover & 64 & 1.9 & 0.4 & 54.4 & 10.9 & $\begin{array}{ll}1.7 & 0.3\end{array}$ & 65.7 & 3.2 & 13.5 & 0.8 \\
\hline Rye & 64 & 2.0 & 0.4 & 51.8 & 21.1 & $\begin{array}{ll}1.6 & 0.3\end{array}$ & 68.9 & 8.7 & 13.4 & 0.8 \\
\hline Clover & 79 & 4.0 & 2.8 & 71.7 & 46.9 & $2.6 \quad 1.4$ & 84.2 & 16.4 & 16.3 & 1.7 \\
\hline Rye & 79 & 3.8 & 1.3 & 59.2 & 21.9 & 3.11 .2 & 94.2 & 6.7 & 17.1 & 0.8 \\
\hline Clover & 85 & 6.2 & 2.0 & 103.0 & 34.7 & 3.71 .0 & 98.3 & 8.9 & 18.4 & 1.2 \\
\hline Rye & 85 & 6.3 & 2.9 & 105.2 & 79.6 & $\begin{array}{ll}3.1 & 0.9\end{array}$ & 100.5 & 9.1 & 18.4 & 1.2 \\
\hline Clover & 99 & 10.5 & 2.5 & 134.8 & 49.9 & 3.91 .2 & 119.0 & 9.0 & 21.1 & 1.8 \\
\hline Rye & 99 & 8.5 & 4.3 & 101.1 & 58.3 & 3.01 .4 & 97.9 & 18.6 & 19.6 & 1.7 \\
\hline Clover & 120 & 21.1 & 4.6 & 182.2 & 73.5 & 5.31 .5 & 124.3 & 8.2 & 22.2 & 1.0 \\
\hline Rye & 120 & 21.1 & 6.8 & 146.8 & 73.9 & 4.81 .9 & 115.1 & 11.8 & 21.1 & 2.0 \\
\hline
\end{tabular}

a DAP is days after planting.

b Biomass, Total N, and LAI values are geometric means.

${ }^{c}$ Values in parentheses indicate $\pm 95 \%$ confidence limits.

* Indicates significant difference between clover and rye values at the same DAP based on T-test at $\alpha \leq 0.10$.
Table 2 Mean above-ground biomass, total $\mathrm{N}$ accumulated in above-ground biomass, plant height and number of nodes at three dates during the 1998 cotton-growing season following crimson clover and rye cover crops.

\begin{tabular}{|c|c|c|c|c|c|c|c|c|c|}
\hline \multirow[t]{3}{*}{ Cover Crop } & \multirow[t]{3}{*}{$\mathrm{DAP}^{\mathrm{a}}$} & \multicolumn{2}{|c|}{ Biomass $^{\mathrm{b}}$} & \multicolumn{2}{|c|}{ Total $\mathrm{N}^{\mathrm{b}}$} & \multicolumn{2}{|c|}{ Height } & \multicolumn{2}{|c|}{ Nodes } \\
\hline & & $\mathrm{Mg} \mathrm{h}$ & $\mathrm{aa}^{-1}$ & $\mathrm{~kg} \mathrm{ha}^{-}$ & & $\mathrm{cm}$ & & \# & \\
\hline & & & STD & & std & & std & & Std \\
\hline Crimson clover & 55 & 4.0 & 0.9 & 74.4 & 20.2 & 53.5 & 5.2 & 14.1 & 1.2 \\
\hline Rye & & 4.5 & 0.7 & 73.5 & 17.9 & 59.5 & 3.2 & 15.0 & 0.7 \\
\hline Crimson clover & 77 & 5.5 & 1.6 & 136.3 & 41.2 & 75.4 & 5.4 & 17.2 & 3.2 \\
\hline Rye & & 6.1 & 1.4 & 148.9 & 37.3 & 75.6 & 5.3 & 17.9 & 2.1 \\
\hline Crimson clover & 132 & 11.3 & 3.3 & 213.2 & 67.5 & 83.9 & 10.0 & 22.5 & 1.3 \\
\hline Rye & & 9.4 & 3.2 & 157.0 & 56.5 & 79.7 & 5.3 & 20.7 & 1.2 \\
\hline
\end{tabular}

a DAP is days after planting.

${ }^{\mathrm{b}}$ Biomass, Total N, and LAI values are geometric means.

${ }^{c}$ Values in parentheses indicate $\pm 95 \%$ confidence limits.

* Indicates significant difference between clover and rye values at the same DAP based on T-test at $\alpha \leq 0.10$. 